\title{
On Contact Heat Transfer with Melting* (The Melting on the Inner Surface of a Horizontal Cylindrical Tube)
}

\author{
Akio SAITO**, Yoshio UTAKA** \\ and Yasuji TOKIHIRO***
}

\begin{abstract}
The direct contact melting phenomena on a cylindrical heat transfer surface, set horizontally, is discussed analytically and experimentally. The basic equations for the thin liquid layer between the heat transfer surface and the phase change boundary are derived and normalized. Then, numerical calculations are performed over wide parameter ranges to determine the liquid layer thickness distribution and the heat flux distribution on the heat transfer surface. The experiments are also performed using the water as the phase change material. The analytical and experimental results concerning the average heat flux at the heat transfer surface are found to agree well with each other. The exact solution for the heat flux distribution on the heat transfer surface is shown for the case of a small Stefan number, where the heat transfer through the liquid layer is approximated by the pure conduction.
\end{abstract}

Key Words: Thermal Engineering, Phase Change, Heat Transfer with Melting, Thermal Energy Storage Capsule, Phase Change Material

\section{Introduction}

In a direct contact melting phenomena, where a solid is pressed on a heat transfer surface whose temperature is higher than the melting point and melts at the contacting boundary, the phase change rate is considerably high, because the latent heat of fusion is supplied from the heat transfer surface through a very thin liquid layer produced by the phase change.

In the storage process (the melting process of a phase change material) of a cylindrical latent heat thermal energy storage capsule set horizontally, as shown in the former paper, for example, the solid phase drops onto the capsule wall by the solid-liquid density difference, and direct contact melting occurs between the solid bottom and the high temperature wall $^{(1)}$. In such a situation, the liquid phase produced

* Received 7th June, 1987. Paper No. 86-0690 A

* Faculty of Engineering, Tokyo Institute of Technology, 2-12-1 Ookayama, Meguro-ku, Tokyo, 152, Japan

*** Mitsubishi Rayon Co., 20-1 Miyuki-cho, Otake, 73906, Japan by the phase change flows out through a very thin liquid layer between the capsule wall and the phase change boundary, and the static pressure within the liquid layer, acting on the phase change boundary, balances with the contacting force of the solid.

In the former reports ${ }^{(2)(3)}$, the authors showed analytical and experimental results concerning the direct contact melting heat transfer of a cylindrical solid which was pressed on a horizontal flat heat transfer surface of uniform temperature, keeping its axis perpendicular to the surface. In those reports, basic equations controlling the phenomena were first derived and normalized to show that the nondimensional heat flux at the heat transfer surface was a function of only two parameters, namely, the Stefan number and a nondimensional contacting force. A numerical method to solve the above basic equations, where the liquid layer thickness distribution was also calculated at the same time, was proposed and the results of the numerical analysis were shown for a wide range of parameters. Then, experiments were performed, using ice and $N$-octadecane as phase change materials, under various combinations of the heat transfer surface temperature, the contacting area 
and the contacting force. The analytical and experimental results agreed quite well with each other. Moreover, a formula correlating the nondimensional heat flux with the Stefan number and the non-dimensional contacting force was proposed ${ }^{(4)}$, covering wide parameter ranges, including the situation where the liquid flow at the melting boundary had a significant effect on the phenomena. Using the formula, the transient characteristics of a latent heat thermal energy storage apparatus utilizing the direct contact melting phenomena was discussed generally.

Sparrow and Myrum ${ }^{(5)}$ measured the solid shape variation within an inclined cylindrical capsule through a direct contact melting process and discussed the relation between the melting rate and the contacting situation. Moallemi and Viskanta ${ }^{(6)}$ investigated a direct contact melting system experimentally and analytically, where a cylindrical heat source was set horizontally in a phase change material and was lowered, melting the surrounding material. Bareiss and $\mathrm{Beer}^{(7)}$ analyzed a direct contact melting process within a horizontal hollow cylinder, assuming the heat transfer mechanism in the liquid layer as the pure conduction and ignoring the liquid velocity effect at the phase change boundary.

In this paper, the direct contact melting phenomena on a hollow cylinder inner surface set horizontally is discussed experimentally and analytically. The phenomena is analyzed by precise numerical calculations, and the heat transfer characteristics of a direct contact melting phenomena on a curved surface is clarified, showing also the condition where the phenomena is controlled by pure conduction through the liquid layer.

\section{Nomenclature}

$a:$ thermal diffusivity $\mathrm{m}^{2} / \mathrm{s}$

$c:$ specific heat $\mathrm{J} /(\mathrm{kg} \cdot \mathrm{K})$

$F_{0}$ : contacting force per unit axial length $\mathrm{N} / \mathrm{m}$

$g:$ acceleration of gravity $\mathrm{m} / \mathrm{s}^{2}$

$h:$ liquid layer thickness (Fig. 1) $\mathrm{m}$

$h_{0}$ : liquid layer thickness at $x=0 \mathrm{~m}$

$k:$ thermal conductivity $\mathrm{W} /(\mathrm{m} \cdot \mathrm{K})$

$L$ : latent heat of fusion $\mathrm{J} / \mathrm{kg}$

$P$ : static pressure $\mathrm{Pa}$

$P_{F}:$ mean contact pressure based on the wetted perimeter $=F_{0} /\left(2 x_{0}\right) \quad \mathrm{Pa}$

$P_{A}$ : mean contact pressure based on the width of a solid phase $=F_{0} /(2 R \sin \Theta) \quad \mathrm{Pa}$

$q:$ local heat flux on a heat transfer surface $\mathrm{W} / \mathrm{m}^{2}$

$\bar{q}:$ mean heat flux on a heat transfer surface $\mathrm{W} / \mathrm{m}^{2}$

$R:$ radius of curvature of a heat transfer surface
(Fig. 1) m

$T$ : temperature $\mathrm{K}$

$T_{m}$ : melting point $\mathrm{K}$

$T_{w}$ : heat transfer surface temperature $\mathrm{K}$

$\Delta T \equiv T_{w}-T_{m}$

$u, v:$ velocities in $x, y$ directions $\mathrm{m} / \mathrm{s}$

$V_{0}$ : melting rate in the gravitational direction $\mathrm{m} / \mathrm{s}$

$x, y:$ co-ordinates (Fig. 2), $x=R \theta \quad \mathrm{m}$

$x_{0}:$ value of $x$ at the end of a liquid layer, $=R \Theta$ $\mathrm{m}$

$\theta:$ angle (Fig. 1)

$\Theta:$ value of $\theta$ at the end of a liquid layer (Fig. 1)

$\mu$ : coefficient of viscosity $\mathrm{Pa} \cdot \mathrm{s}$

$\rho:$ density $\mathrm{kg} / \mathrm{m}^{3}$

$C^{*}=\left(\partial T^{*} / \partial y^{*}\right)_{y^{*}=h^{*}}$

$C_{0}^{*}=C^{*} / \cos \theta=\rho L x_{0} V_{0} /(k \cdot \Delta T)$

$\bar{C}^{*}=-\int_{0}^{1} C^{*} d x^{*}$

$h^{*}=h / x_{0}$

$h_{0}^{*}=h_{0} / x_{0}$

$P^{*}=P / P_{F}$

$q^{*}=x_{0} q /(k \cdot \Delta T)$

$\bar{q}^{*}=x_{0} \bar{q} /(k \cdot \Delta T)$

$S t_{e}=c \Delta T / L$

$T^{*}=\left(T-T_{m}\right) / \Delta T$

$u^{*}=\rho L x_{0} u /(k \cdot \Delta T)$

$v^{*}=\rho L x_{0} v /(k \cdot \Delta T)$

$x^{*}=x / x_{0}$

$y^{*}=y / x_{0}$

\section{Analysis}

2. 1 Basic equations and boundary conditions

A direct contact melting system as shown in Fig. 1 is considered, where a phase change material is pressed on the inner surface of a hollow cylinder heat transfer surface of radius $R$, kept at a constant tem.

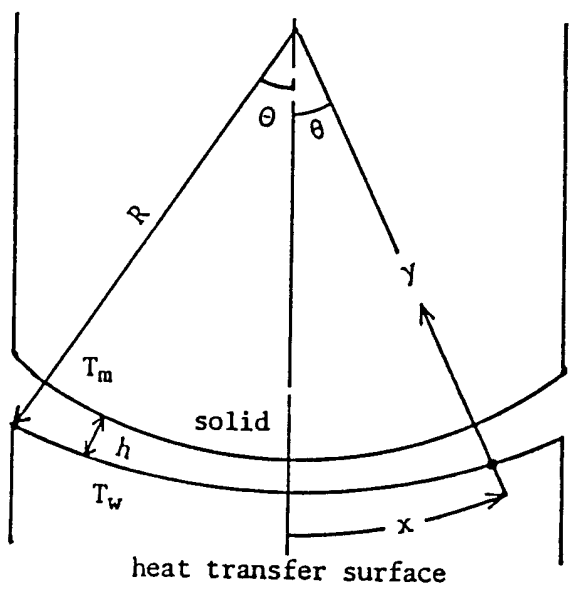

Fig. 1 Coordinate system 
perature $T_{w}$ higher than the melting point $T_{m}$, by a constant force $F_{0}$. The axis of the cylinder is set horizontally, and the system is symmetric concerning the direction of gravity. If the system is in the steady state, the shape of the phase change boundary at the bottom of the solid is unchanged by time, and the melting rate of the solid in the direction of gravity is uniform and constant over the heat transfer surface.

Nondimensional basic equations for the liquid layer between the solid bottom and the heat transfer surface are derived as follows, omitting the small terms under the consideration that the layer is very thin.

Continuity equation:

$$
\frac{\partial u^{*}}{\partial x^{*}}+\frac{\partial v^{*}}{\partial y^{*}}=0
$$

Momentum equation:

$$
\begin{aligned}
& \frac{\partial P^{*}}{\partial x^{*}}=\frac{\mu a}{P_{F} x_{0}^{2}} \frac{c \Delta T}{L} \frac{\partial^{2} u^{*}}{\partial y^{* 2}}-\frac{x_{0}}{P_{F}} \rho g \sin \theta \\
& \frac{\partial P^{*}}{\partial y^{*}}=-\frac{x_{0}}{P_{F}} \rho g \cos \theta
\end{aligned}
$$

Energy equation:

$$
u^{*} \frac{\partial T^{*}}{\partial x^{*}}+v^{*} \frac{\partial T^{*}}{\partial y^{*}}=\frac{L}{c \Delta T} \frac{\partial^{2} T^{*}}{\partial y^{* 2}}
$$

In the above equations, nondimensional values are defined as in the nomenclature. As shown in Fig. 1, $x$ and $y$ are axes in the directions along the cylindrical inner surface and perpendicular to the surface, respectively. Symbols $u, v$ mean the velocities in the $x, y$ directions, and $h(x)$ is the liquid layer thickness in the $y$ direction as a function of $x . V_{0}$ is the melting rate in the direction of gravity. Mean pressure $P_{F}$ is defined as $P_{F}=F_{0} /\left(2 x_{0}\right)$.

Next, boundary conditions are as follows.

On the heat transfer surface:

$$
y^{*}=0 ; u^{*}=0, \quad v^{*}=0, \quad T^{*}=1
$$

At the solid-liquid phase change boundary :

$$
\begin{aligned}
& y^{*}=h^{*} ; u^{*}=0, \quad T^{*}=0 \\
& v^{*}=\left(\partial T^{*} / \partial y^{*}\right)_{y^{*}=h^{*}}=C_{0}^{*} \cos \theta
\end{aligned}
$$

On the plane of symmetry:

$$
x^{*}=0 ; u^{*}=0
$$

At the end of the solid-liquid phase change boundary :

$$
x^{*}=1: P^{*}=0
$$

The balance of the contacting force and the static pressure is expressed by the following relation, where $\Theta=x_{0} / R$.

$$
\int_{0}^{\theta} P^{*} \cos \theta d \theta=\Theta
$$

The following equation for nondimensional heat flux on the heat transfer surface is also obtained.

$$
q^{*}=\frac{x_{0}}{k \Delta T} q=-\left(\frac{\partial T^{*}}{\partial y^{*}}\right)_{y^{*}=0}
$$

It is clear from the above equations that non. dimensional mean heat flux on the heat transfer surface $\bar{q}^{*}$ depends only on four nondimensional parameters : $c \Delta T / L, P_{F} x_{0}^{2} /(\mu \cdot a), x_{0} \rho g / P_{F}$ and $\Theta$.

\subsection{Dependencies of $u^{*}, v^{*}$ and $d P^{*} / d x^{*}$ on $x^{*}$ and $y^{*}$}

In this section, equations $(1) \sim(4)$ are transfor. med into relations which are easier for numerical calculations, considering boundary conditions (5) $\sim(9)$.

Integrating Eq. ( 3 ) by $y^{*}$, the following equation is obtained.

$$
P^{*}=C_{1}\left(x^{*}\right)-\frac{x_{0} \rho g y^{*}}{P_{F}} \cos \theta
$$

The above equation is substituted into Eq. (2), and the resulting equation is integrated twice by $y^{*}$, considering boundary conditions $(5)(6)$, to obtain the following equation.

$$
\begin{aligned}
u^{*} & =\frac{1}{2}\left(\frac{L}{c \Delta T}\right)\left(\frac{P_{F} x_{0}^{2}}{\mu a}\right) y^{*}\left(y^{*}-h^{*}\right) \\
& \times\left[\frac{d C_{1}}{d x^{*}}+\frac{x_{0} \rho g}{P_{F}}\left\{\frac{\Theta}{3}\left(y^{*}+h^{*}\right)+1\right\} \sin \theta\right]
\end{aligned}
$$

The above equation is simplified, considering $1 \gg$ $\Theta\left(y^{*}+h^{*}\right) / 3$.

$$
\begin{aligned}
u^{*} & \fallingdotseq \frac{1}{2}\left(\frac{L}{c \Delta T}\right)\left(\frac{P_{F} x_{0}^{2}}{\mu a}\right) y^{*}\left(y^{*}-h^{*}\right) \\
& \times\left\{\frac{d C_{1}}{d x^{*}}+\frac{x_{0} \rho g}{P_{F}} \sin \theta\right\}
\end{aligned}
$$

The mass balance in the interval $0 \sim \theta$ is expressed as

$$
\int_{0}^{h *} u^{*} d y^{*}=-\left(\frac{L}{c \Delta T}\right)\left(\frac{V_{0} R}{a}\right) \sin \theta
$$

and $d C_{1} / d x^{*}$ is obtained from the above equation and Eq. (10).

$$
\begin{aligned}
& \frac{d C_{1}}{d x^{*}}=12\left(\frac{c \Delta T}{L}\right)\left(\frac{\mu a}{P_{F} x_{0}^{2}}\right)\left(\frac{C_{0}^{*}}{\Theta}\right) \frac{\sin \theta}{h^{* 3}} \\
& -\frac{x_{0} \rho g}{P_{F}} \sin \theta
\end{aligned}
$$

From Eqs. (10) and (11), the following equation is obtained.

$$
u^{*}=\frac{6 C_{0}^{*}}{\Theta} y^{*}\left(y^{*}-h^{*}\right) \frac{\sin \theta}{h^{* 3}}
$$

The following equation is also derived from Eqs. (1) (12).

$$
\begin{aligned}
& v^{*}=-C_{0}^{*} \frac{y^{* 2}}{h^{* 4}}\left\{h^{*} \cos \theta \cdot\left(2 y^{*}-3 h^{*}\right)\right. \\
&\left.+\frac{6}{\Theta} \sin \theta \cdot\left(h^{*}-y^{*}\right) \frac{d h^{*}}{d x^{*}}\right\}
\end{aligned}
$$

Then, Eq. ( 2 ) is transformed by Eq. (12).

$$
\begin{aligned}
\frac{d P^{*}}{d x^{*}} & =12\left(\frac{c \Delta T}{L}\right)\left(\frac{\mu a}{P_{F} x_{0}^{2}}\right)\left(\frac{C_{0}^{*}}{\Theta}\right) \frac{\sin \theta}{h^{* 3}} \\
-\frac{x_{0} \rho g}{P_{F}} \sin \theta &
\end{aligned}
$$

In Eqs. $(12) \sim(14)$, the relation $\theta=\left(x_{0} / R\right) x^{*}$ is satisfied.

\section{3 Numerical calculation}

When nondimensional parameters $c \Delta T / L, P_{F} x_{0}^{2} /$ 
$(\mu \cdot a), x_{0} \rho g / P_{F}$ and $\Theta$ are given, solutions for $u^{*}, v^{*}$ and $T^{*}$ as functions of $x^{*}$ and $y^{*}$, and solutions for $P^{*}$ and $h^{*}$ as functions of $x^{*}$, which satisfy Eqs. (4), (9), (12) - (14) and boundary conditions $(5),(6),(6)^{\prime}$, (8) simultaneously, are decided. The process of the finite difference calculation is explained below, dividing the liquid layer into small elements along the $x^{*}$, $y^{*}$ axes. One of the difficulties of the following numerical calculation is that the liquid layer thickness $h^{*}$ is also an unknown function of $x^{*}$, and the liquid layer thickness distribution is decided through an iteration process, being modified until the solutions satisfy all the above equations. In the explanation below, $\Delta x^{*}$ and $\Delta y^{*}$ denote finite differences in $x^{*}$ and $y^{*}$ directions, respectively. Symbols $C_{i}^{*} h_{i}{ }^{*}, P_{i}^{*}, u_{i j}^{*}, v_{i j}^{*}$ and $T_{i j}^{*}$ are defined as constant $C^{*}$ liquid layer thickness $h^{*}$, and pressure $P^{*}$ at place $x_{i}^{*}=i \Delta x^{*}(i=1 \sim 20)$, and as velocities $u^{*}, v^{*}$, and temperature $T^{*}$ at place $x_{i}^{*}, y_{j}^{*}$ $=j \Delta y^{*}(j=1 \sim 40)$, respectively.

The numerical calculation is performed by the following process shown in the flow-chart of Fig. 2.

(1) The liquid layer thickness distribution $h^{*}\left(x^{*}\right)$ and the nondimensional heat flux at the phase change boundary, $C_{0}^{*}$, are assumed.

(2) From Eqs. (4), (12) (14), distributions of $P_{i}^{*}, u_{i j}^{*}, v_{i j}^{*}$ and $T_{i j}^{*}$ which satisfy boundary conditions

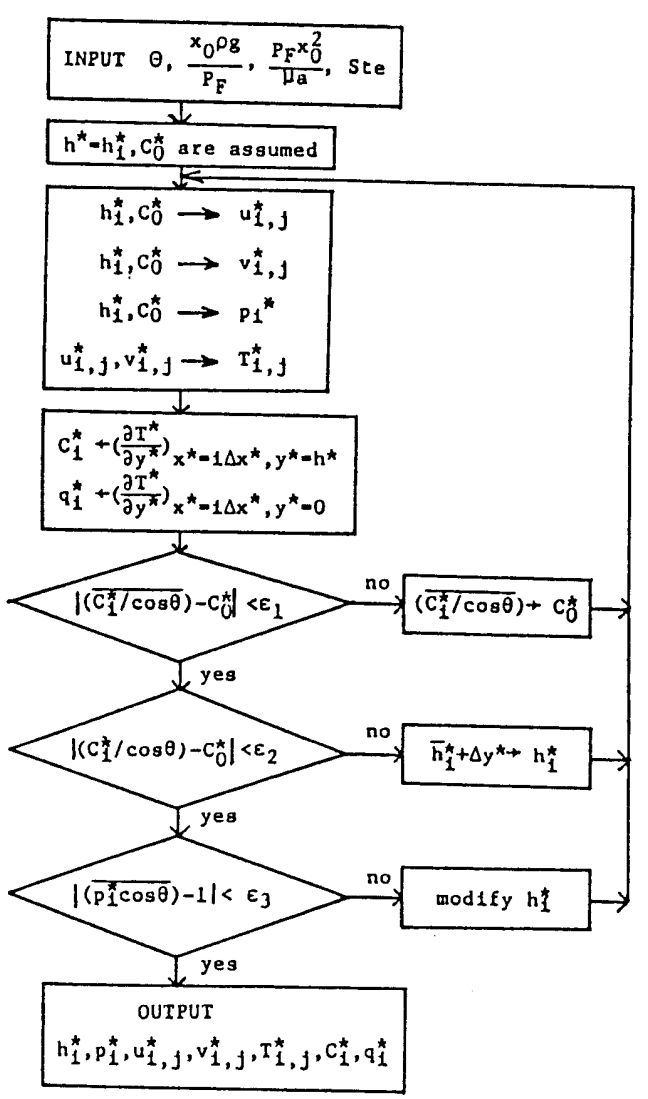

Fig. 2 Flow-chart
( 5 ) ( 6 ) ( 8 ), are calculated. Then, heat flux distributions at the phase change boundary, $C_{i}^{*}$, and at the heat transfer surface, $q_{i}^{*}$, are decided from the temperature distribution.

(3) If $P_{i}^{*}$ and $T_{i j}^{*}$, thus calculated, satisfy boundary conditions $(6)^{\prime}(9)$, it is concluded that all solutions are obtained, and that the calculation is finished. If boundary conditions ( 6$)^{\prime}$ and (9) are not satisfied, the following modifications are done.

(3-1) If the mean value $\overline{C_{i}^{*} / \cos \theta}$ of $C_{i}^{*} / \cos \theta$ which is calculated from $C_{i}^{*}$, obtained in step (2), is not equal to the assumed value $C_{0}^{*}$, the value $C_{0}^{*}$ is replaced by the new value $\overline{C_{i}^{*} / \cos \theta}$, and the calculation goes back to step (2).

(3-2) If the value $C_{i}^{*} / \cos \theta$ calculated from $C_{i}^{*}$, obtained in step (2), is not uniform over the phase change boundary, the liquid layer thickness distribution is modified, and the calculation goes back to step (2).

(3-3) If the mean value of the pressure component $P_{i}^{*} \cos \theta$, obtained in step ( 2$)$, is not unity, the mean value of the liquid layer thickness is modified, and the calculation goes back to step (2).

By repeating the above steps $(1) \sim(3)$, the numerical solutions are obtained.

\section{Experiments}

\section{1 Experimental apparatus}

Experiments were performed using ice as the phase change material. As shown in Fig. 3, the upper surface of a copper block was used as the heat transfer surface, having a radius of curvature of $R=17.5$ $\mathrm{mm}$, a length of $240 \mathrm{~mm}$ and a contacting angle of $\Theta$ $=\pi / 3$. On the bottom three surfaces of the heat transfer block, nickel-chrome foil heaters of with a thickness of $30 \mu \mathrm{m}$ (resistance $5.3 \Omega$ ) were attached, inserting thin mica plates between the block and the heaters, and were pressed by insulating plates (silicone rubber plates with a thickness of $1.5 \mathrm{~mm}$ ) and

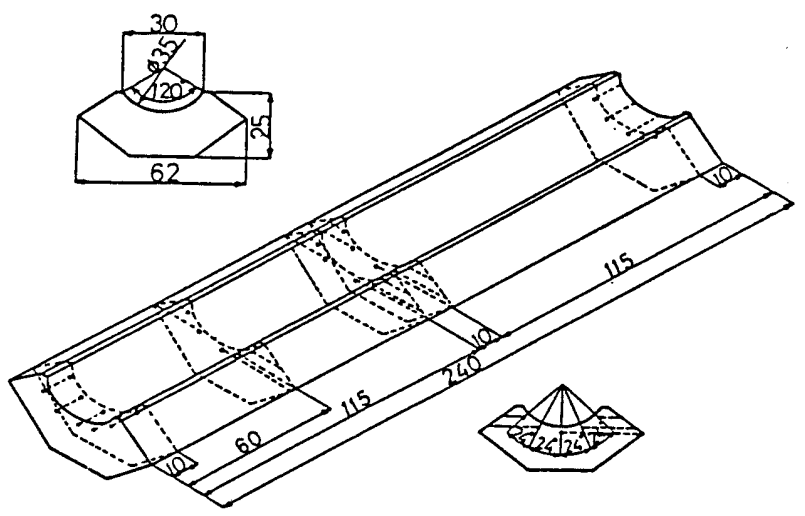

Fig. 3 Heat transfer block and thermocouple locations 
bakelite plates. By controlling the output of the heaters, the heat transfer block was kept at a uniform and constant temperature. The thermal resistance between the foil heater and the block was adjusted by bolts which fixed the bakelite plate to the block. In the block, twenty-five $\mathrm{Cu}$-Co thermocouples with a diameter of $0.2 \mathrm{~mm}$ were put at places shown in Fig. 3 at a depth of $1 \mathrm{~mm}$ from the surface. The radial and longitudinal temperature distributions of the block were measured using these thermocouples.

The whole apparatus is shown in Fig. 4. Over the heat transfer block mentioned above, acrylic sidewall plates were attached, and an ice block, having almost the same width as the gap between the sidewalls, was inserted downward, and was subjected to direct contact melting on the heat transfer block. The water produced by the phase change was exhausted through narrow gaps at the bottom of the acrylic walls. The melting rate of the ice was determined from the displacement of the ice top, measured by a dial gauge. The contacting force on the phase change boundary was determined from the weight of the ice and the attachments.

In the experiment, an ice block was carefully prepared so as to eliminate the formation of bubbles within it. An ice block, having almost no bubbles, was inserted in a container, and the gap was filled with boiled water. Then the container was chilled to freeze the water. To decrease the effect of sensible heat of ice, the ice block was heated very slowly in a refrigerator until the ice block had uniform temperature distribution near the melting point.

\section{2 Experimental method}

Experiments were performed, setting the mean contact pressure $P_{F}$ at about $414 \mathrm{~Pa}$ or $4140 \mathrm{~Pa}$, and keeping the heat transfer surface at various temperatures between $0.4 \sim 8.2^{\circ} \mathrm{C}$. The contact pressure of $414 \mathrm{~Pa}$ corresponds to the case where an ice block of

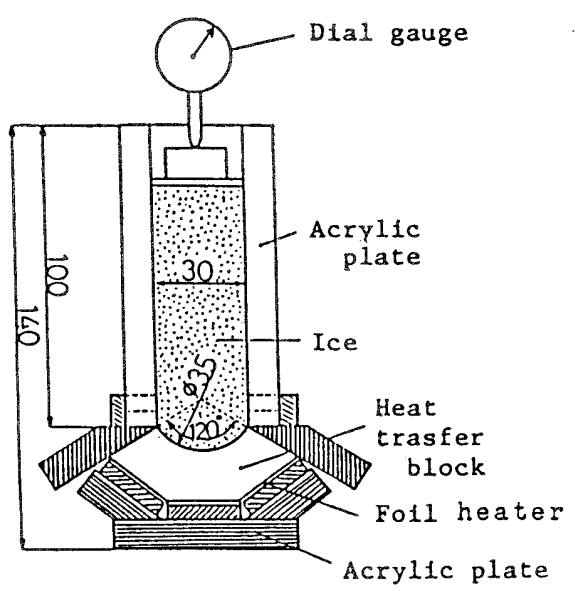

Fig. 4 Experimental apparatus
$6 \mathrm{~cm}$ in height is placed on a heat transfer surface and contacts, being pressed by its own weight.

The heat transfer surface was set horizontally, and a degassed ice block was pressed on it, supplying heat by heaters. When the shape of the bottom of the ice block fitted the heat transfer surface concave and the system arrived at the steady state, the heat transfer surface temperature, the heat flux at the surface, the melting rate and the contact pressure were measured.

\section{Results and Discussion}

\section{1 Comparison between experimental and ana- lytical results}

In chapter 2., the mean contact pressure based on the wetted perimeter, $P_{F}$, was used to define nondimensional parameters. But it is rational to use the component of the gravitational direction for understanding the actual phenomena. In this section, therefore, the apparent contact pressure defined as the contact force per unit project area of the gravitational direction, $P_{A}=F_{0} /(2 R \sin \Theta)$, is used. The relation between $P_{A}$ and $P_{F}$ is expressed as $P_{A}=x_{0} P_{F} /(R \sin$ $\Theta)$.

The measured relations of nondimensional mean heat flux on the heat transfer surface, $\bar{q}^{*}$, versus the Stefan number are shown in Fig. 5 by symbols $\bigcirc$ and $\triangle$, setting nondimensional contact pressure $P_{A} x_{0}^{2} /(\mu$. a) at $7.14 \times 10^{8}$ and $7.14 \times 10^{9}$, respectively. For both cases, the angle is set at $\Theta=\pi / 3$, but the nondimensional parameter $x_{0} \rho g / P_{A}$ corresponds to 0.313 and 0.0313 , respectively, because the experiments are performed under given contact forces. The solid lines in Fig. 5 are the result of numerical calculations explained in chapter 2. Experimental and analytical results agree quite well with each other, and the numerical method proposed in this paper is confirmed

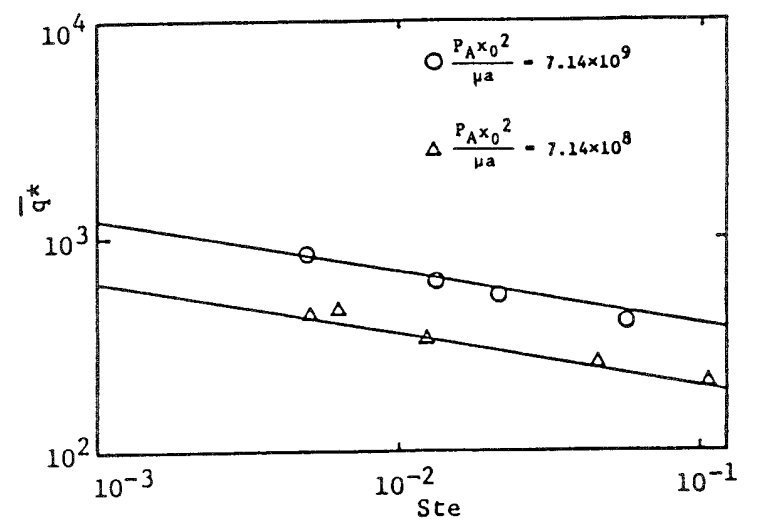

Fig. 5 Comparison between experimental and analytical results 
to be reasonable.

\section{2 Discussions on the heat transfer character-} istics through numerical calculations

Numerical calculations by the method proposed in this paper were done to clarify the details of the direct contact melting phenomena, which is difficult to discuss experimentally. Figure 6 shows the variations of nondimensional mean heat fluxes on the heat transfer surface, $\bar{q}^{*}$, and at the phase change boundary, $\bar{C}^{*}$, versus the Stefan number Ste for the cases where nondimensional parameter $P_{A} x_{0}^{2} /(\mu \cdot a)$ is $10^{8}, 10^{9}$ and $10^{10}\left(x_{0} \rho g / P_{A}=2.23,0.223\right.$ and 0.0223$)$, respectively. It is clear from Fig. 6 that $\bar{q}^{*}$ and $\bar{C}^{*}$ coincide in the range of Ste $<0.1$, showing straight lines of a constant gradient. Over such a range, the effect of velocity at the phase change boundary is negligible, and the phenomena is controlled by heat conduction through the liquid layer. The fact that the inequality $\bar{q}^{*}>\bar{C}^{*}$ appears in the range Ste $>0.1$ is due to the sensible heat of the exhausted liquid layer.

In Fig. 7, the relation between nondimensional liquid layer thickness $h_{0}{ }^{*}$ at $x^{*}=0$ and the Stefan number Ste is shown for the same experimental condi-

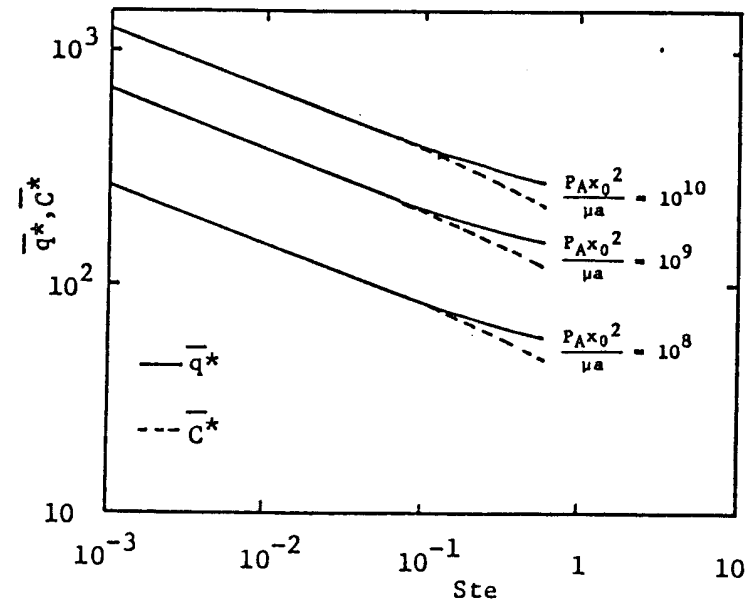

Fig. 6 Relation among Ste, $\bar{q}^{*}$ and $\bar{C}^{*}$

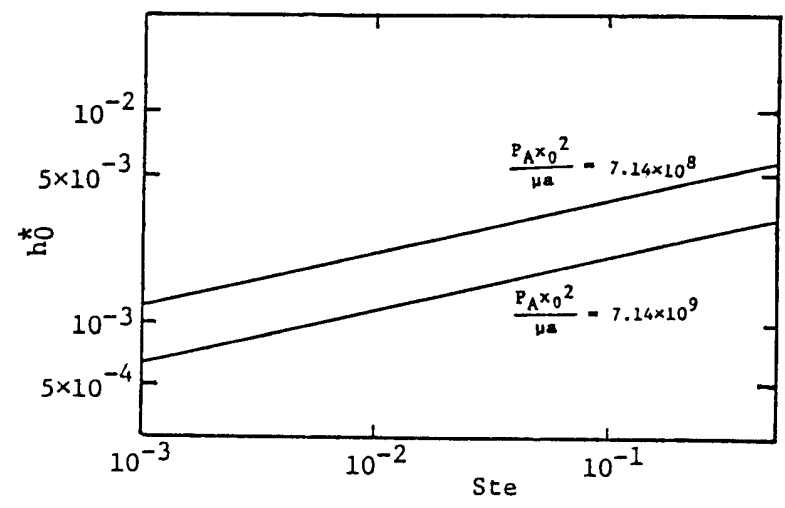

Fig. 7 Relation between Ste and $h_{0}^{*}$ tions as in Fig. 5. The thickness $h_{0}{ }^{*}$ increases almost linearly with Ste in the co-ordinates of logarithmic scales. If the nondimensional value $h_{0}{ }^{*}=10^{-3}$ is calculated in terms of the actual thickness of the experimental conditions of this paper, $h_{0}$ is approximately $18.3 \mu \mathrm{m}$ This result means that the liquid layer is very thin in the case of direct contact melting, and that the heat flux is considerably high.

Figures 8(a) (b) show the distributions of nondimensional liquid layer thickness $h^{*}$ for the same condition as in Fig. 7. The distributions of nondimensional local heat flux $q^{*}$ for the same condition as in Fig. 7 are also shown in Figs. 9(a)(b). From the results of Figs. 8(a)(b) and Figs. 9(a)(b), the following relations are satisfied almost completely.

$$
\begin{aligned}
& h^{*}=h_{0}^{*} / \cos \theta \\
& q^{*}=1 / h^{*}
\end{aligned}
$$

These results coincide with the above mentioned fact that the phenomena is controlled by heat conduc. tion through the liquid layer for Ste $<0.1$.

\section{3 Analytical solution for the range where the} phenomena is controlled by heat conduction

Under the condition where Eqs.(15)(16) are satisfied, Eq. (14) is easily integrated, and $h_{0}{ }^{*}$ is solved

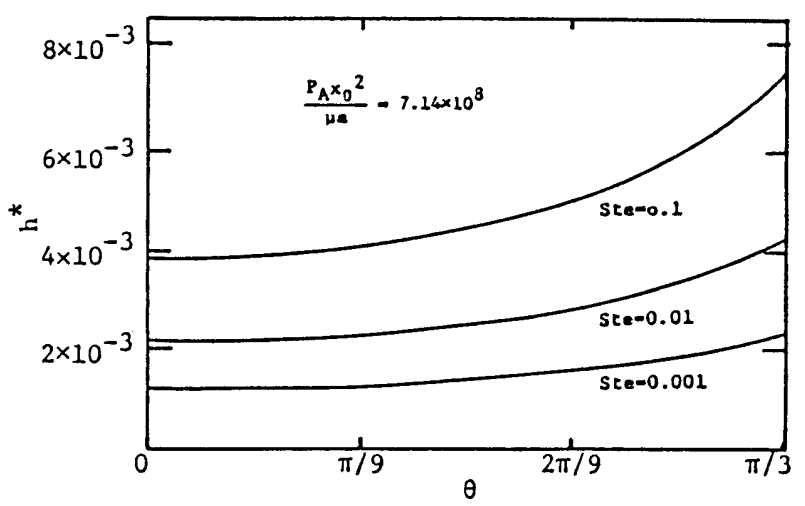

(a) Distribution for $P_{A} x_{0}^{2} /(\mu \cdot a)=7.14 \times 10^{8}$

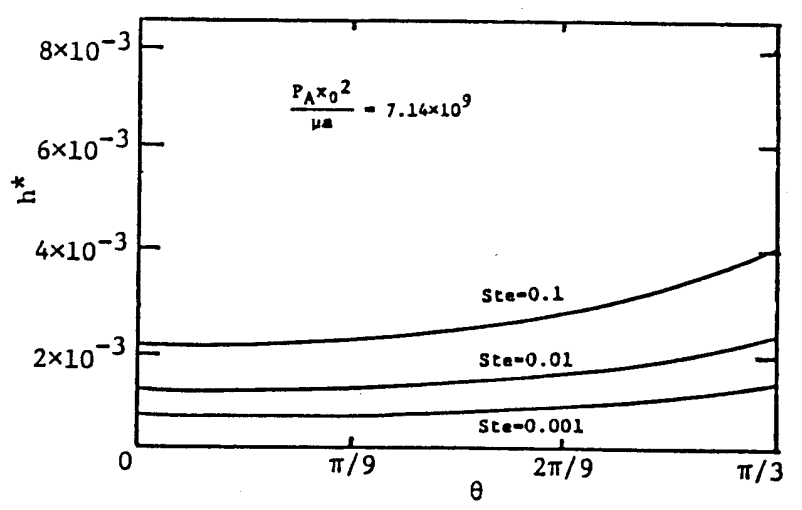

(b) Distribution for $P_{A} x_{0}^{2} /(\mu \cdot a)=7.14 \times 10^{9}$

Fig. 8 Distribution of $h^{*}$ 


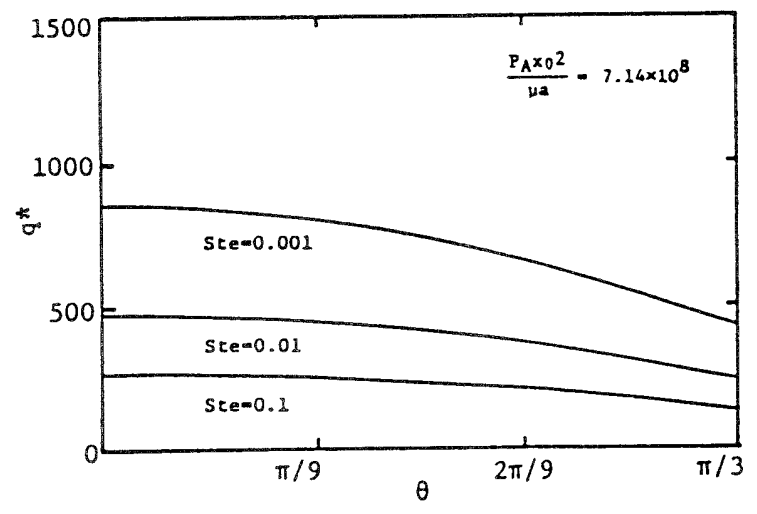

(a) Distribution for $P_{A} x_{0}^{2} /(\mu \cdot a)=7.14 \times 10^{8}$

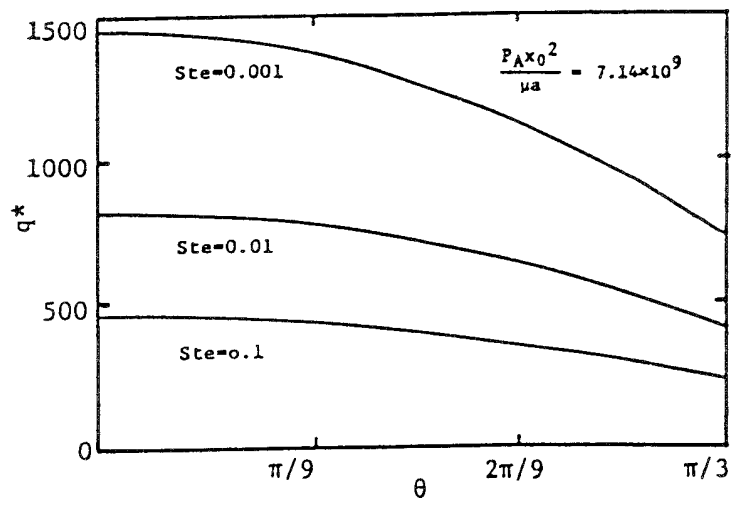

(b) Distribution for $P_{A} x_{0}^{2} /(\mu \cdot a)=7.14 \times 10^{9}$

Fig. 9 Distribution of $q^{*}$

as a function of Ste, $P_{A} x_{0}^{2} /(\mu \cdot a), x_{0} \rho g / P_{A}$ and $\Theta$ by the process shown in the Supplement. The solution is shown below.

$$
\begin{aligned}
h_{0}^{*} & =\left[\left\{\frac{3 \text { Ste }}{\Theta \sin \Theta} /\left(\frac{P_{A} x_{0}^{2}}{\mu a}\right)\right\}\right. \\
& \times\left(\frac{1}{80} \sin 5 \Theta+\frac{5}{48} \sin 3 \Theta+\frac{5}{8} \sin \Theta\right. \\
& \left.-\cos ^{4} \Theta \sin \Theta\right) /\left\{\Theta-\frac{1}{2}\left(\frac{x_{0} \rho g}{P_{A}}\right)\right. \\
& \left.\left.\times\left(\frac{\Theta}{\sin \Theta}-\cos \Theta\right)\right\}\right]^{1 / 4}
\end{aligned}
$$

Using Eqs. (15)-(17), distributions of $h^{*}$ and $q^{*}$ are obtained. Therefore, nondimensional mean heat flux $\bar{q}^{*}$ is given by the following equation.

$$
\bar{q}^{*}=\frac{\sin \Theta}{\Theta h_{0}^{*}}
$$

\section{Conclusion}

Analytical and experimental discussions were conducted for the steady state direct contact melting system, where a phase change material was pressed by a constant force on a cylindrical horizontal heat transfer surface with a uniform temperature. The following conclusions were reached.

(1) Basic equations and boundary conditions, which describe the heat transfer phenomena within a thin liquid layer between the heat transfer surface and the phase change boundary, were derived and normalized. Then, it was clarified that the nondimensional mean heat flux on the heat transfer surface, $\bar{q}^{*}$, is a function of only $c \Delta T / L, P_{F} x_{0}^{2} /(\mu \cdot a), x_{0} \rho g / P_{F}$ and $\Theta$.

(2) The above basic equations were transformed into simpler forms for numerical calculations, and a numerical method, where the distribution of the liquid layer thickness is also calculated at the same time, was proposed.

(3) Experiments using ice as the phase change material were performed to show that the results of the above numerical method agree well with the experimental results.

(4) Numerical calculations were also done for parameters of various values. It was shown that the effect of melted liquid velocity at the phase change boundary is significant in the range of Ste $>0.1$. The system was simplified for the condition of Ste $<0.1$, and exact solutions of nondimensional liquid layer thickness $h^{*}$ and nondimensional mean heat flux $\bar{q}^{*}$ were shown.

Finally, the authors wish to express their appreciation to Mr. T. Fujikake, who was a student of Tokyo Institute of Technology, for his kind cooperation.

\section{Supplement}

From Eqs. (14) (15), the following equation is derived.

$$
\begin{aligned}
& \frac{d P^{*}}{d x^{*}}=12 \operatorname{Ste}\left(\frac{\mu a}{P_{F} x_{0}^{2}}\right)\left(\frac{C_{0}^{*}}{\Theta}\right) \frac{\sin \theta \cos ^{3} \theta}{h_{0}^{* 3}} \\
& -\frac{x_{0} \rho g}{P_{F}} \sin \theta
\end{aligned}
$$

Integrating the above equation and considering the boundary conditions of Eq. ( 8 ), the pressure distribution is obtained as :

$$
\begin{aligned}
P^{*} & =-3 \operatorname{Ste}\left(\frac{\mu a}{P_{F} x_{0}^{2}}\right) \frac{C_{0}^{*}}{\Theta^{2} h_{0}^{* 3}}\left(\cos ^{4} \theta-\cos ^{4} \Theta\right) \\
& +\left(\frac{x_{0} \rho g}{P_{F}}\right) \frac{1}{\Theta}(\cos \theta-\cos \Theta)
\end{aligned}
$$

Eliminating the pressure term $P^{*}$ from Eqs. (9) (19) and again eliminating the term $C_{0}^{*}$ from the resulting equation by the following relation derived from Eq. (16),

$$
-C_{0}^{*} \cos \theta=q^{*}=1 / h^{*}
$$

the final solution is obtained as Eq. (17).

\section{References}

(1) Katayama, K., Saito Akio, Saito Akihiro, Utaka, Y., Matsui, H., Maekawa, H. and Saifulula, A., Solar Energy, Vol.27, No. 2 (1981), p. 91. 
(2) Saito, A., Utaka, Y., Akiyoshi, M. and Katayama, K., Bull. JSME, Vol. 28, No. 240 (1985), p. 1142.

( 3 ) Saito, A., Utaka, Y., Akiyoshi, M. and Katayama, K., Bull. JSME, Vol. 28, No. 242 (1985), p. 1703.

(4) Saito, A., Utaka, Y., Shinoda, K. and Katayama, K., Bull. JSME, Vol. 29, No. 255 (1986), p. 2946.
(5) Sparrow, E. M. and Myrum, T. A., J. Heat Transf., Vol. 107, No. 3 (1985), p. 533.

(6) Moallemi, M. K. and Viskanta, R., J . Heat Transf., Vol. 107, No. 2 (1985), p. 451.

( 7 ) Bareiss, M. and Beer, H., Int. J. Heat \& Mass Transf., Vol. 27, No. 5 (1984), p. 739. 